\title{
Polyploidy in deep and shallow evolutionary times: from ancient cotton, middle aged tobacco to recently formed monkey-flowers
}

\author{
Ales Kovarik $^{1} \cdot$ Višnja Besendorfer $^{2} \cdot$ Miroslav Plohl $^{3} \cdot$ Eric Schranz $^{4}$
}

Received: 21 September 2017 / Accepted: 22 September 2017 / Published online: 3 October 2017

(C) Springer-Verlag GmbH Austria 2017

Keywords Biodiversity $\cdot$ Biological databases $\cdot$ Ecology $\cdot$ Evolution · Hybridization · Genetic variation · Plant . Polyploidy

Topics aimed for understanding evolution of life on earth bridge science, culture and even philosophy. It has been well established that interspecies hybridization (merger of two or more different genomes), polyploidization (chromosome multiplication) and allopolyploidy, which combines both events, are major drivers of plant and animal genome evolution. There is no doubt that human civilization is entirely dependent on polyploid species providing food, fodder, cotton (clothes) and biofuels. Most of them are relatively recent polyploids that formed within last few million years. One can therefore ask important questions, such as how stable are the crop species' genomes and phenotypes over time? Will they be able to cope with global climate change? How about their agricultural traits? Will they be able to meet constant demand for food production? Based on what we know about the genome dynamics of polyploid species, we can envisage that that future corn fields will not be the same as in these days. Hence, understanding

Ales Kovarik

kovarik@ibp.cz

1 Institute of Biophysics, Czech Academy of Sciences, Brno CZ61265, Czech Republic

2 Department of Biology, University of Zagreb, Zagreb, Croatia

3 Laboratory for Structure and Function of Heterochromatin, Ruđer Bošković Institute, Zagreb, Croatia

4 Department of Biosystematics, University of Wageningen, Wageningen, Netherlands polyploidy in deep and shallow evolutionary times is challenging, timely and even strategic aim.

The aforementioned facts have motivated researchers to organize International Conferences on Polyploidy, Biodiversity and Hybridization (ICPHB). The history of these successful meetings goes back to the second half of the last century when the first international conference on polyploidy was held at the Washington University in St. Louis during May 1979. In this meeting, wide spread relevance of polyploidy in nature was suggested (Lewis 2004). Discoveries of several allopolyploid systems that formed just within past centuries were among the highlights of the second ICPHB, held in London in 2003 (Bennett 2004). A good message for the ecologists is that despite overall reduction in biodiversity, newly formed allopolyploid species and hybrids potentially balance species extinction. By the time of the 2009 San Malo (France) ICPHB, it had become clear that modern sequencing technologies coupled with advanced bioinformatics will be key factors influencing methodology and future progress in the field (Ainouche and Jenczewski 2010). For example, utilizing these stateof-the-art genomic methods revealed that all seed plants have experienced several whole genome duplications, the oldest dated before the split of angiosperm and gymnosperm lineages. Thus, polyploidy is not a dead end of evolution but instead is an inevitable part of it. The 2011 Prague (Czech Republic) conference, already held in the post-genomic era, was the first to combine plant and animal polyploidy research. Here the scientific community acknowledged the increasing awareness of the potential role of polyploidy and hybridization in adaptation and invasiveness (Mable 2013). The purpose of the present special issue is to pay tribute to the 2016 ICPHB held in Rovinj (Croatia), which hosted more than 200 participants from 22 countries. The following paragraph has no ambition to 
review individually this remarkable set of papers, but we would like to preface their main achievements.

Several papers addressed a disputed issue of relationship between polyploidy and speciation. Clarkson et al. (2017 in this issue), by studying polyploidy in Nicotiana, observed a several million year lag phase between initial polyploidy event(s) and species radiation. One can ask whether this mysterious period apparently reflecting ongoing micro- and macromutations in young polyploids provocatively evokes partially discredited Goldschmidt's hypothesis of "hopeful monsters". The second Nicotiana paper, by Dodsworth et al. (2017 in this issue), describes a new phylogenomic pipeline based on quantification of repeats constituting major structural components of chromosomes. Such an approach has illuminated complicated phylogenetic relationships among ancient Nicotiana allopolyploids.

Two papers addressed the issue of genetic interactions between the co-resident subgenomes in allopolyploid species. Grover et al. (2017 in this issue) took a wholegenomic approach to analyse two Gossypium (cotton) allotetraploids. Genetic diversity in both species is low, having been influenced by both the polyploid bottleneck and a domestication bottleneck (for cultivated accessions), but with a directional bias in homoeolog diversity favouring the same genome in both allopolyploids. Lunerová et al. (2017 in this issue) analysed single nucleotide polymorphisms (SNPs) in Nicotiana tabacum (tobacco) by high throughput methods, showing that much of the parental variation at rDNA loci has been removed by interlocus gene conversion. The authors hypothesize that such a process may eliminate pseudogenes that accumulated in the diploid parental genomes over time.

The amount of epigenetic diversity within and among populations is important to assess ecological consequences of polyploidy. Carvalho et al. (2017 in this issue) studied expression variation in natural populations in the context of divergent and reticulate polyploid speciation. Using ecologically powerful Spartina system, the authors demonstrated interpopulation differences in expression patterns of several genes including those involved in abiotic stress response. The paper by Lorenzo et al. (2017 in this issue) reviews the current literature on epigenetic regulatory pathways involved in plant development. Other ecologically significant contributions have been nicely commented by Rob Denton in his Molecular ecologist blog: http://www. molecularecologist.com/2016/05/genomes-on-the-beachthe-international-conference-on-polyploidy-hybridizationand-biodiversity/.

Two papers deal with issues of reproduction of polyploid species. Vallejo-Marín et al. (2017 in this issue), using well-controlled interpopulation crosses of Mimulus peregrines (monkey-flower), observed statistically significant reduction in pollen viability in these hybrids, implying that gene flow may be limited between allopolyploid populations of independent origin. Formation of reproductive barriers between cytotypically differentiated populations of Ranunculus kuepferii is the topic of the Schinkel et al. (2017 in this issue) paper. The authors analysed large number of individuals in natural populations of providing evidence that unreduced egg cell formation represents first step towards apomixis.

A considerable wealth of information on various polyploidy and biodiversity linked topics has accumulated in the literature. There is no wonder that three papers in this issue describe novel or updated internet databases which will be useful for data mining and further statistical analyses. Dressler et al. (2017 in this issue) assembled an online portal of German flora displaying georeferenced and validated herbarium specimens of apomictic plants. Paule et al. (2017 in this issue) created a database of chromosome counts and genome sizes of the German flora. Finally, Vitales et al. (2017 in this issue) present an updated rDNA loci database displaying information about the genome sequencing projects and telomeric domains.

The 2016 ICPHB is now a history, while it foreshadows the promise of continued progress to be revealed at the next meeting, in Ghent, Belgium, scheduled for 2019. We look forward to this meeting, as most researchers would agree that no electronic communication could replace or surpass the personal communication via the oral presentation, discussions at posters, fruitful chats at coffee breaks, pubs and even debates on a sandy beach, that characterized this meeting in Rovinj.

The ICPHB was organized by the University of Zagreb Croatia and the Croatian Biological Society. General chairman of the conference was Višnja Bessendorfer, University of Zagreb, Croatia, to whom we would like to express my special thanks for her indefatigable support. Many thanks belong to the international steering committee and scientific board and to all referees who have accepted to make reviews of all these papers in a really short time. Many thanks also belong to the Plant Systematics and Evolution team, mainly its Editors-in-Chief, Marcus Koch, Martin Lysak and Karol Marhold. A very special thanks goes to Barbora Šingliarová and Katarína Olšavská for their excellent assistance in publishing this special issue of the journal.

\section{References}

Ainouche ML, Jenczewski E (2010) Focus on polyploidy. New Phytol 186:1-4. doi:10.1111/j.1469-8137.2010.03215.x

Bennett MD (2004) Perspectives on polyploidy in plantsancient and neo. Biol J Linn Soc 82:411-423. doi:10.1111/j.1095-8312.2004.00328.x 
Clarkson JJ, Dodsworth S, Chase MW (2017) Time-calibrated phylogenetic trees establish a lag between polyploidisation and diversification in Nicotiana (Solanaceae). Pl Syst Evol 303:1-12. doi:10.1007/s00606-017-1416-9

de Carvalho JF, Boutte J, Bourdaud P, Chelaifa H, Ainouche K, Salomon A, Ainouche M (2017) Gene expression variation in natural populations of hexaploid and allododecaploid Spartina species (Poaceae). Pl Syst Evol 303:1-19. doi:10.1007/ s00606-017-1446-3

Dodsworth S, Jang T-S, Struebig M, Chase MW, Weiss-Schneeweiss H, Leitch AR (2017) Genome-wide repeat dynamics reflect phylogenetic distance in closely related allotetraploid Nicotiana (Solanaceae). Pl Syst Evol 303:1-8. doi:10.1007/ s00606-016-1356-9

Dressler S, Gregor T, Hellwig FH, Korsch H, Wesche K, Wesenberg J, Ritz CM (2017) Comprehensive-and reliable-a new online portal of critical plant taxa in German. P1 Syst Evol 303:1-5. doi:10.1007/s00606-017-1419-6

Grover CE, Gallagher JP, Szadkowski EP, Page JT, Gore MA, Udall JA, Wendel JF (2017) Nucleotide diversity in the two co-resident genomes of allopolyploid cotton. Pl Syst Evol 303:1-22. doi:10.1007/s00606-017-1411-1

Lewis WH (2004) Preface. Biol J Linn Soc 82:409. doi:10.1111/j.1095-8312.2004.00353.x

Lorenzo JLR, Hobza R, Vyskot B (2017) Epigenetic control of reproductive development. Pl Syst Evol 303:1-10. doi:10.1007/ s00606-016-1377-4
Lunerová J, Renny-Byfield S, Matyášek R, Leitch A, Kovařík A (2017) Concerted evolution rapidly eliminates sequence variation in rDNA coding regions but not in intergenic spacers in Nicotiana tabacum allotetraploid. Pl Syst Evol 303:1-18. doi:10.1007/s00606-017-1442-7

Mable BK (2013) Polyploids and hybrids in changing environments: winners or losers in the struggle for adaptation? Heredity 110:95-96. doi:10.1038/hdy.2012.105

Paule J, Gregor T, Schmidt M, Gerstner E-M, Dersch G, Dressler S, Wesche K, Zizka G (2017) Chromosome numbers of the flora of Germany - a new online database of georeferenced chromosome counts and flow cytometric ploidy estimates. Pl Syst Evol 303:1-7. doi:10.1007/s00606-016-1362-y

Schinkel CCF, Kirchheimer B, Dullinger S, Geelen D, De Storme N, Hörandl E (2017) Pathways to polyploidy: indications of a female triploid bridge in the alpine species Ranunculus kuepferi (Ranunculaceae). Pl Syst Evol 303:1-16. doi:10.1007/ s00606-017-1435-6

Vallejo-Marín M, Quenu M, Ritchie S, Meeus S (2017) Partial interfertility between independently originated populations of the neo-allopolyploid Mimulus peregrinus. Pl Syst Evol 303:1-12. doi:10.1007/s00606-017-1426-7

Vitales D, D’Ambrosio U, Gálvez F, Kovařík A, Garcia S (2017) Third release of the plant rDNA database with updated content and information on telomere composition and sequenced plant genomes. Pl Syst Evol 303:1-7. doi:10.1007/s00606-017-1440-9 